\title{
Central odontogenic fibroma of the maxilla: a case report with immunohistochemical study
}

\author{
Mitsunobu Kishino', Miki Ishibashi, ${ }^{1,}$, Hidehiko Koizumi ${ }^{2}$, Sunao Sato ${ }^{1}$, Shumei Murakami ${ }^{3}$, \\ Yuzo Ogawa ${ }^{1}$, Mikihiko Kogo ${ }^{2}$, Satoru Toyosawa ${ }^{1}$
}

${ }^{1}$ Department of Oral Pathology, Osaka University Graduate School of Dentistry, Osaka, Japan

${ }^{2}$ Department of Oral \& Maxillofacial Surgery 1, Osaka University Graduate School of Dentistry, Osaka, Japan

${ }^{3}$ Department of Oral \& Maxillofacial Radiology, Osaka University Graduate School of Dentistry, Osaka, Japan

Abstract: A rare case of central odontogenic fibroma of the maxilla is presented. A 29-yearold man was admitted because of movable right lateral incisor and dull pain. Panoramic radiograph showed a well-demarcated unilocular radiolucent lesion in the right anterior region of the maxilla. The enucleated tumor was a well-defined firm mass with a solid yellowish-white appearance. Histologically, the tumor was composed of minimally cellular collagenous connective tissue with myxoid matrix. Small epithelial islands were scanty, scattered and inactive appearing in the connective tissue. Immunohistochemically, the fibroblastic cells were positive for vimentin, and the epithelial islands were positive for cytokeratin 14 (CK14) and CK19, which are markers of odontogenic epithelium. From these findings, the tumor was diagnosed as an epithelium-poor type of central odontogenic fibroma. No recurrence has been noted 14 months after surgery.

[Oral Med Pathol 2009; 14: 29-32 doi: 10.3353/omp.14.29]

Key words: central odontogenic fibroma, cytokeratin, epithelium-poor type, maxilla, odontogenic epithelium

Correspondence: Mitsunobu Kishino, Department of Oral Pathology, Osaka University Graduate School of Dentistry, 1-8 Yamadaoka, Suita, Osaka 565-0871, Japan

Phone: +81-6-6879-2892, Fax: +81-6-6879-2895, E-mail: mkishino@dent.osaka-u.ac.jp

\section{Introduction}

Central odontogenic fibroma $(\mathrm{COF})$ is a rare and benign neoplasm, which comprises $1.5 \%$ of all intraosseous odontogenic tumors (1). COF is considered to be derived from the mesenchymal tissue of odontogenic origin such as dental follicle or periodontal ligament (2). According to Gardner's review (3), COF is mainly identified in lesions with two different histological patterns. The first, known as epithelium-poor type (simple type), contains connective tissue resembling a dental follicle with scattered remnants of odontogenic epithelium. The second epithelium-rich type (WHO or complex type) contains fibrous connective tissue with varying amounts of odontogenic epithelium. Furthermore, dysplastic dentin- or cementum-like materials may sometimes be found (3-4). The lesions can occur at intraosseous or gingival areas, and the term 'odontogenic fibroma' is applied to both intraosseous (central) and gingival (peripheral) lesions (5). The clinical and radiographic features of COF have been well documented, but the histological aspects have generated controversy (3-4, 6-7).

In this case report, we describe clinical and histological features of a case of COF in the anterior maxillary region and discuss the immunohistochemical findings of it, especially cytokeratin (CK) 14 and CK19 as markers of odontogenic epithelium.

\section{Case report}

A 29-year-old man was admitted to our hospital because of movable right lateral incisor and dull pain of the upper jaw. Panoramic radiograph showed a well-demarcated

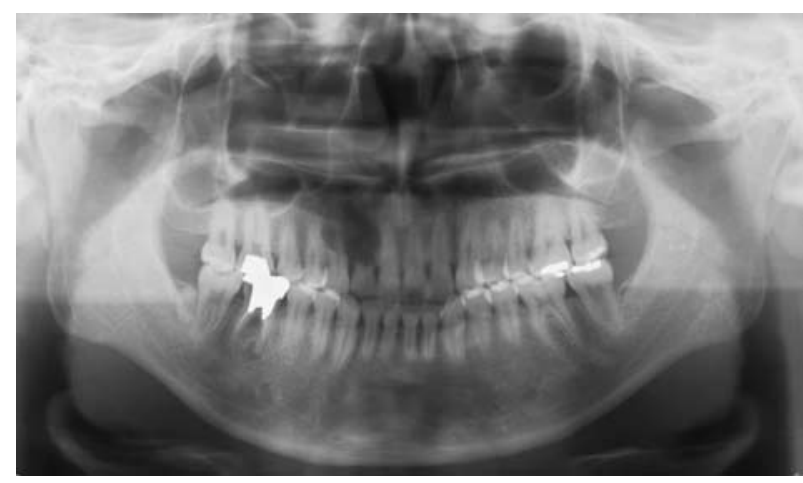

Fig. 1. Panoramic radiograph of central odontogenic fibroma (COF) arising in the right incisor region of the maxilla of a 29 -year-old man. The lesion was represented as a well-demarcated unilocular radiolucency with resorption of the incisor tooth root. 


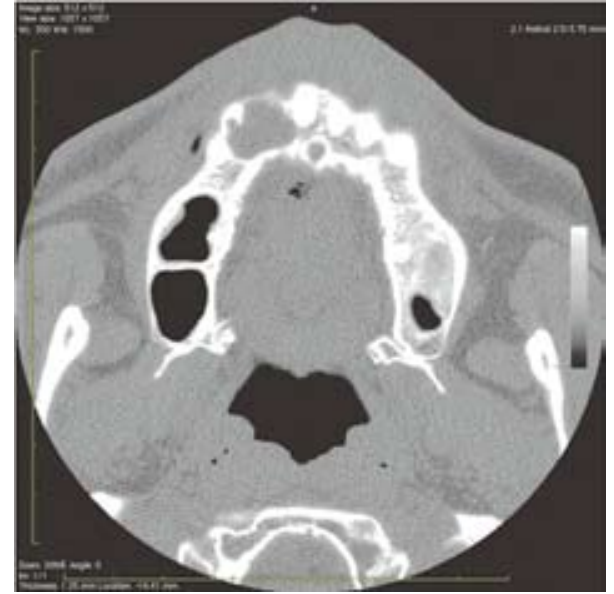

Fig. 2. Bone target computed tomography (CT) of COF of the maxilla. CT showed a slight labial bulge and thin cortical bone in the right incisor region of the maxilla.

unilocular radiolucent lesion between the right lateral incisor and the right canine of the upper jaw with distinct root resorption (Fig. 1). Bone target CT showed a slight labial bulge with thin cortical bone (Fig. 2). The patient underwent extraction of the lateral incisor and incisional biopsy of the lesion, which revealed a benign fibrous tumor. The tumor was enucleated with extraction of the right canine. No exposure of the tumor out of the cortical bone was found. The tumor was a well-defined firm mass with a solid yellowish-white appearance (Fig. 3). There have been no signs of recurrence approximately 14 months after the operation.



Fig. 3. Gross finding of surgically-removed COF of the maxilla. Macroscopically, the enucleated tumor with the extracted right canine was a well-defined firm mass with a solid yellowish-white appearance.

The tissues obtained by surgery were fixed in $10 \%$ phosphate-buffered neutral formalin followed by paraffin embedding. Histological sections from paraffin-embedded blocks were stained with hematoxylin and eosin (HE). Microscopic examination showed a less cellular fibrous connective tissue characterized by densely packed interwoven bundles of collagen with myxoid matrix (Fig. 4a). The connective tissue contained fibroblastic cells, which elongated with fusiform or wavy appearing nuclei (Fig. 4b). Inactive-appearing epithelial islands were scattered in the connective tissue (Fig. 4c). The epithelial cells were small
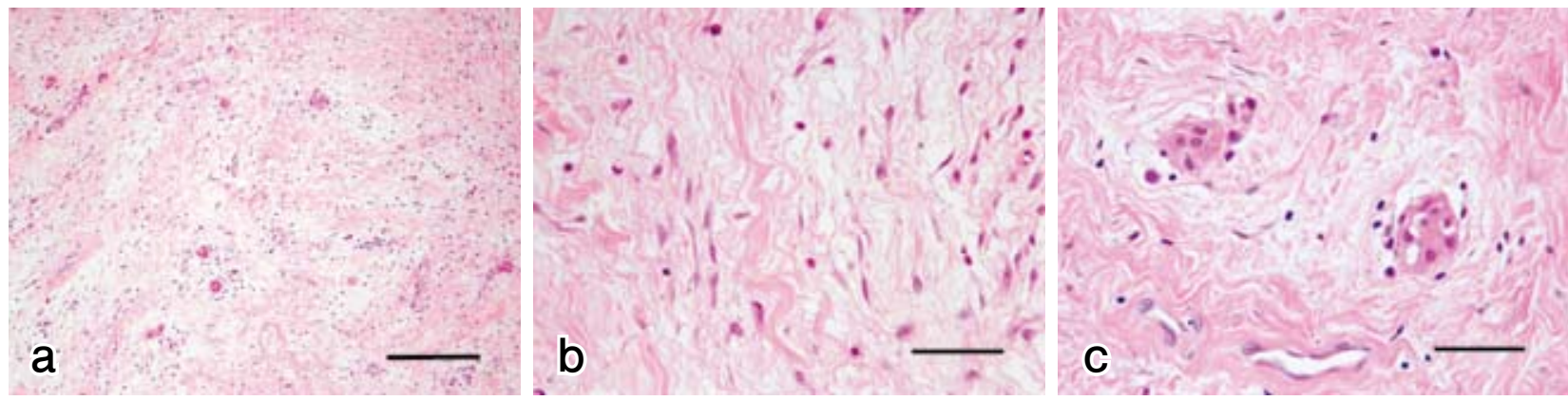

Fig. 4. Histological findings of COF of the maxilla. (a-c) HE stain, bars $=200 \mu \mathrm{m}(\mathrm{a}), 50 \mu \mathrm{m}(\mathrm{b}, \mathrm{c}$ ). The tumor was composed of minimally cellular collagenous connective tissue with myxoid matrix, and small epithelial islands were scattered (a). The connective tissue contained fibroblastic cells, which elongated with fusiform or wavy appearing nuclei (b). The epithelial cells were small and round with a vacuolated cytoplasm $(\mathbf{c})$.

Table 1. Primary antibodies used in immunohistochemistry for central odontogenic fibroma

\begin{tabular}{lllc}
\hline antibody & clone & source & dilution \\
\hline multi-cytokeratin & AE1/AE3 & Dako & pre-diluted \\
cytokeratin 13 & KS-1A3 & Novocastra & $1: 200$ \\
cytokeratin 14 & LL002 & Novocastra & $1: 80$ \\
cytokeratin 18 & DC-10 & Novocastra & $1: 40$ \\
cytokeratin 19 & RCK108 & Dako & $1: 100$ \\
vimentin & V9 & Dako & pre-diluted \\
S-100 protein & polyclonal & Dako & pre-diluted \\
alpha-smooth muscle actin & 1A4 & Dako & $1: 200$ \\
\hline
\end{tabular}



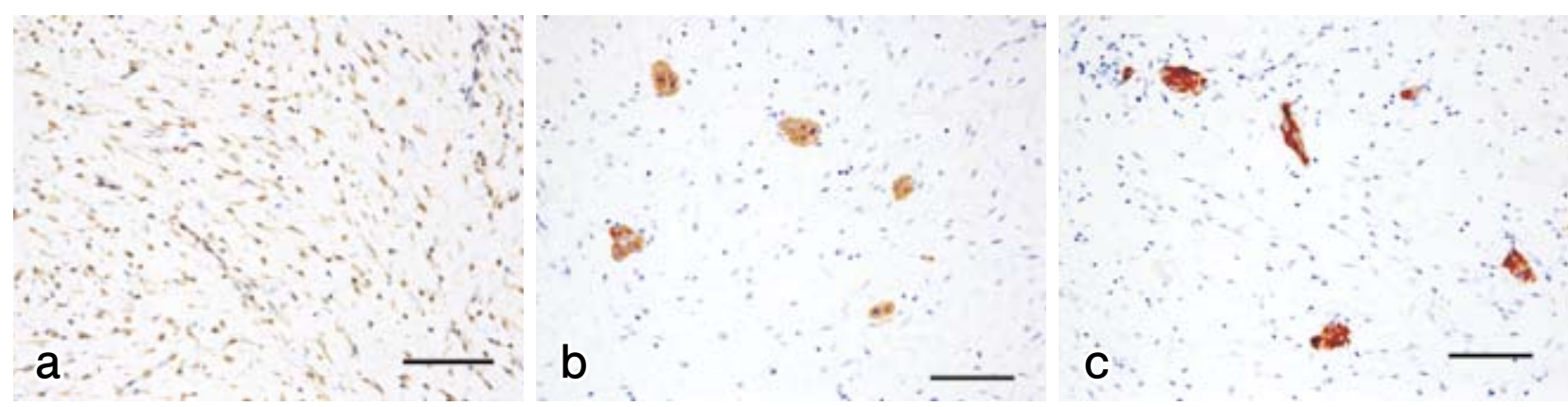

Fig. 5. Immunohistochemical findings of COF of the maxilla. Immunoperoxidase stains for vimentin (a), CK14 (b), and CK19 (c), hematoxylin counterstain, bars $=100 \mu \mathrm{m}$. The fibroblastic cells were positive for vimentin (a). The epithelial cells showed positive stainings for CK14 (b) and CK19 (c).

and round, and often had a vacuolated cytoplasm. Dysplastic dentin- or cementum-like materials were not present in the lesion.

Immunohistochemical staining was performed on the serially prepared paraffin-embedded sections by EnVision+ system (Dako, Glostrup, Denmark). Antigen retrieval was carried out in citrate buffer $(0.01 \mathrm{~mol} / \mathrm{l}, \mathrm{pH} 7.0)$ at $121^{\circ} \mathrm{C}$ for 10 minutes by an autoclave. Antibodies used in this report are listed in Table 1. The fibroblastic cells were positive for vimentin (Fig. 5a), but negative for alpha-smooth muscle actin and S-100 protein. The epithelial islands showed positive staining for multi-cytokeratin (AE1/AE3), CK14 (Fig. 5b) and CK19 (Fig. 5c), but negative for CK13 and CK18. Therefore, the epithelial islands were demonstrated to be odontogenic epithelium.

From these histological and immunohistochemical findings, the tumor was diagnosed as a simple type of COF.

\section{Discussion}

The COF is a rare, benign, mesenchymal neoplasm in the jawbone, which comprises $1.5 \%$ of all intraosseous odontogenic tumors in the series of Buchner et al. (1). According to the reviews of the literature on $\operatorname{COF}(1,7-10)$, the higher frequency of the lesion is in the fourth to fifth decades. It is more common in women than in men. There is no significant predilection for the location of it either in the maxilla or the mandible. The most common locations are the posterior region of the mandible and the anterior region of the maxilla. Radiographically, the COFs show unilocular or multilocular radiolucency with well-defined, thinly corticated margins. Root divergence or resorption is often present. Bony expansion without cortical perforation is present in enlarged lesions. These general clinical and radiological features are mostly consistent with the characteristics of our case.

Histologically, the COF is composed of mature fibrous tissue and the presence of varying amounts of inactivelooking odontogenic epithelial nests and cords (2). At present, the term COF is applied to two histological types of lesion: the epithelium-poor type (simple type) and the epithelium-rich type (complex or WHO type) (2-3). The epithelium-poor type of COF consists of collagenous connective tissue with myxoid matrix, which resembles a dental follicle. Scattered remnants of inactive-looking odontogenic epithelium appear as small irregular islands and cords. The epithelium-rich type of COF consists of more cellular fibroblastic connective tissue with conspicuous islands or strands of inactive-looking odontogenic epithelium. Our case was diagnosed as an epithelium-poor type of COF from the histological findings of minimally cellular collagenous connective tissue with scanty small epithelial islands. It is suggested that the epithelium-rich type of COF arises from periodontal ligament because of the histological similarity between tumor connective tissue component and periodontal ligament, while the epithelium-poor type is derived from dental follicle because of the histological similarity between tumor connective tissue component and dental follicle $(3,7)$. In an earlier study (11), hyperplastic dental follicles were interpreted as COF because of the histological similarities between these two lesions. We have to be careful of this misdiagnosis, considering the apparent clinical-radiologic correlation with the histopathological findings.

COFs are tumors of odontogenic origin $(2-3,7)$. Previous reports show that the epithelial components of the odontogenic tumors immunohistochemically express various kinds of CKs-CK7, 8, 13, 14, 18 and 19- and then almost all epithelial cells are positive for CK14 and CK19 (12-15). Thus, CK14 and CK19 are considered as markers of odontogenic epithelium. De Lima et al show that the epithelial islands of COF-like lesion are positive for multicytokeratin (AE1/AE3) and CK19 (16). In our results, epithelial islands of the tumor, which expressed CK14 and CK19, were demonstrated to be odontogenic in origin. The fibrous tissue was positive for vimentin, but negative for alpha-smooth muscle actin and S-100 protein, which were the markers of smooth muscle and peripheral nerve. These immunohistochemical findings also support that it can be diagnosed as COF.

No recurrence has be noted 14 months after enucleation of the tumor. Enucleation or curettage is recommended as treatment for both types of $\operatorname{COF}(4,7)$. Recurrence is uncommon because the lesion is solid and well circumscribed. It is considered that long-term follow-up of our case is not necessary because of adequate excision of the tumor. 


\section{References}

1. Buchner A, Merrell PW, Carpenter WM. Relative frequency of central odontogenic tumors: a study of 1,088 cases from Northern California and comparison to studies from other parts of the world. J Oral Maxillofac Surg 2006; 64: 1343-52.

2. Philipsen HP, Reichart PA, Sciubba JJ, van der Waal I. Odontogenic fibroma. In: Barnes L, Eveson JW, Reichart P and Sidransky D ed. World Health Organization Classification of Tumours: Pathology and Genetics of Head and Neck Tumours. IARC Press, Lyon. 2005; 315.

3. Gardner DG. Central odontogenic fibroma current concepts. J Oral Pathol Med 1996; 25: 556-61.

4. Dunlap CL. Odontogenic fibroma. Semin Diagn Pathol 1999; 16: 293-6.

5. Daley TD, Wysocki GP. Peripheral odontogenic fibroma. Oral Surg Oral Med Oral Pathol 1994; 78: 329-36.

6. Sciubba JJ, Fantasia JE, Kahn LB. Benign odontogenic tumors. In: Rosai J ed. Tumors and Cysts of the Jaws. Atlas of Tumor Pathology. $3^{\text {rd }}$ series, Fascicle 29. Armed Forces Institute of Pathology, Washington DC. 2001; 105-8.

7. Handlers JP, Abrams AM, Melrose RJ, Danforth R. Central odontogenic fibroma: clinicopathologic features of 19 cases and review of the literature. J Oral Maxillofac Surg 1991; 49: 46-54.

8. Daniels JS. Central odontogenic fibroma of mandible: a case report and review of the literature. Oral Surg Oral Med Oral Pathol Oral Radiol Endod 2004; 98: 295-300.
9. Ikeshima A, Utsunomiya T. Case report of intra-osseous fibroma: a study on odontogenic and desmoplastic fibromas with a review of the literature. J Oral Sci 2005; 47: 149-57.

10. Silva CO, Sallum AW, do Couto-Filho CE, Costa Pereira AA, Hanemann JA, Tatakis DN. Localized gingival enlargement associated with alveolar process expansion: peripheral ossifying fibroma coincident with central odontogenic fibroma. J Periodontol 2007; 78: 1354-9.

11. Bhaskar SN. Oral pathology in the dental office: survey of 20,575 biopsy specimens. J Am Dent Assoc 1968; 76: 761-6.

12. Ong'uti MN, Howells GL, Williams DM. An immunohistochemical study of keratin expression in ameloblastoma from a Kenyan population. Oral Dis 1999; 5: 111-6.

13. Tateyama H, Tada T, Okabe M, Takahashi E, Eimoto T. Differentiate keratin profiles in craniopharyngioma subtypes and ameloblastomas. Pathol Res Pract 2001; 197: 735-42.

14. Crivelini MM, de Araujo VC, de Sousa SO, de Araujo NS. Cytokeratins in epithelia of odontogenic neoplasms. Oral Dis 2003; 9: 1-6.

15. Kishino M, Murakami S, Yuki M, et al. A immunohistochemical study of the peripheral ameloblastoma. Oral Dis 2007; 13: 575-80.

16. de Lima Mde D, de Aquino Xavier FC, Vanti LA, de Lima PS, de Sousa SC. Hybrid central giant cell granuloma and central odontogenic fibroma-like lesion of the mandible. Otolaryngol Head Neck Surg 2008; 139: 867-8. 